\title{
Effects of Binocular Acuity in Visual Impairment Assessment
}

\section{Guangfeng Wu1, Lan Wang1, Baozeng Li , Chao Zai1', Shanyao Zhaor, Hong $\mathrm{CaO}^{2}$}

\author{
${ }^{1}$ Department of Forensic Medicine, Qingdao University Medical College, Qingdao 266071, People's Republic of \\ China \\ *Public Security Bureau of Qingdao \\ ${ }^{2}$ Affiliated Hospital of Qingdao University
}

\begin{abstract}
Objective: To study the correlations and differences between best corrected visual acuity in the better eye (hereinafter referred to as monocular acuity in the better eye) and binocular acuity after both eyes were best corrected separately (hereinafter referred to as binocular acuity), the relationship between both monocular acuity in the better eye and binocular acuity and reading acuity as well as reading speed were discussed in order to investigate the effect of binocular acuity in visual impairment and visual disability assessment. Methods: Two thousand a hundred and fifteen subjects ranging in age from 18 to 40 years old were recruited for the study, whose best corrected monocular acuity were less than or equal to 0.8 , and other visual functions examined were normal. Best corrected visual acuity was measured monocularly and binocularly using Standard Logarithm Distance Charts. Reading visual acuity and reading speed were also determined. Experimental data were statistically analyzed using SPSS19.0 statistical software. Results: Monocular acuity in the better eye and binocular acuity was linear correlation $(r=0.920, P<0.001)$, and the differences of them were statistically significant $(\mathrm{Z}=-23.025, \mathrm{P}<0.05)$; Reading acuity and both monocular acuity in the better eye and binocular acuity were also linear correlation $(r=0.827, P<0.001 ; r=0.862, P<0.001)$; Reading acuity deteriorated with aggravation of visual impairment.There were no differences of reading speed in visual impairment individuals that more than or equal to 0.3 ; However ,reading speed in visual disability (visual acuity was less than 0.3) individuals were slowed down significantly. Conclusions: Both of monocular acuity in the better eye and binocular acuity could estimate visual function preferably. There were also differences between both of them. In view of people are simultaneously using both eyes in daily life, it is suggested that the visual function of binocular acuity was recommended in the evaluation of judicial forensic visual impairment and visual disability.
\end{abstract}

Keywords: Binocular acuity; Visual disability; Visual impairment; Visual function

Central distant visual acuity is short for distant visual acuity or visual acuity, which is an important predictor of visual function and is used clinically to assess visual impairment. ${ }^{1}$ Best corrected visual acuity in the better eye is assessment criteria in the evaluation of judicial forensic visual impairment and visual disability at present in China, also American Social Security Administration to determine disability is visual acuity in the better seeing eye, as a measure of binocular function. ${ }^{2}$ However, both eyes are usually used to perform work, learning and other visual activities, binocular vision is the most common form of visual condition in daily life. Monocular acuity in the better eye is not considered the coordination and the effectiveness in real life with two eyes, it can not represent for the visual acuity in everyday life. Therefore, not only monocular acuity in the better eye should be considered in the evaluation of judicial forensic visual impairment and visual disability, but also the binocular acuity. The relationship between monocular acuity in the better eye and visual function in visual impairment individuals, as well as binocular acuity was studied in this paper. Expected that attention should be paid for binocular acuity and binocular visual function in the evaluation of judicial forensic visual impairment and visual disability in the future, so that the assessment of visual acuity is more scientific and reasonable.

\section{Subjects and Methods 1.1 Subjects}

Two thousand a hundred and fifteen participants ranging in age from 18 to 40 years old (the average age was 34.6) were selected for the study, whose

This article is published under the terms of the Creative Commons Attribution License 4.0

Author(s) retain the copyright of this article. Publication rights with Alkhaer Publications.

Published at: http://www.ijsciences.com/pub/issue/2015-10/

DOI: 10.18483/ijSci.853; Online ISSN: 2305-3925; Print ISSN: 2410-4477 
best corrected visual acuity in the better were less than or equal to 0.8 , other visual functions (visual field, color vision, contrast sensitivity, dark adaptation, eye movement, etc. ) examined were normal. All experimental data were from optometry room of Affiliated Hospital of Qingdao University from May to August in 2015. Including 824 male and 1291 female. The purpose of the experiment were told at first, agreed to participate in this research, and cooperated to complete the whole experimental procedures.

\subsection{Methods and Procedures}

All the experimental data were collected and recorded by the same examiner in optometry room of Affiliated Hospital of Qingdao University. The optometry room was $6 \mathrm{~m}$ length and $4 \mathrm{~m}$ width, interior lighting was provided by $60 \mathrm{~W}$ incandescent lamp, brightness was about 45 lux. The same inspection item should be examined 3 times. Visual acuity and reading acuity were recorded and described by Decimal Records Act, then converted to Five Points Records Act for statistical analysis.

\subsubsection{Visual Acuity Tests}

As part of an extensive psychophysical test battery, visual acuity was examined with Standard Logarithm Distance Charts transilluminated with the Lighthouse Chart Illuminator to a level of approximately 350 (candela) cd $/ \mathrm{m} 2$. Acuity was tested at $5 \mathrm{~m}$.If the participant was unable to read the largest letters on the chart, test distance was reduced to $4 \mathrm{~m}$ and testing repeated. This procedure was repeated until an acuity measure was obtained or the participant failed at a distance of $1 \mathrm{~m}$. If one was still could not identify the largest letters, then tested counting fingers $(\mathrm{CF})$, hand movement (HM) and light perception (LP) or no light perception (NLP) in a dark room.

Acuity was measured binocularly then monocularly (right eye followed by left eye) with the participant's optimal refractive correction.If the acuity was worse than 0.8 with either eye, then a complete subjective refraction was performed using optometry lens worn in a trial frame.

\subsubsection{Tests of Reading Visual Acuity and Reading Speed}

Reading acuity was tested with <Chinese Reading Visual Acuity Chart> (Chenxiao Wang et al. People's Medical Publishing House, 2011) using corrected binocular acuity of subjects. Illuminated with fluorescent lamp, the surface of the text was uniform illumination (350lx) and no reflection. Short passages of text were displayed and the participants read aloud as quickly as they could. Thirteen print sizes were tested ranging from 33 point to 2 point. Each print size was tested 3 times in random order. Reading visual acuity was tested at $40 \mathrm{~cm}$. If the participant was unable to read the largest print sizes on the text, test distance was reduced to $25 \mathrm{~cm}$ and testing repeated. The minimum print size of the text that corresponds to a visual acuity was the final reading acuity,recorded it. Reading speed was also measured at the same time.Those who could not read recorded as 0 .

\subsection{Statistical Methods}

All experimental data were inputted using excel, and analyzed by SPSS19.0 statistical software. Including:Spear-man correlation analysis and non-parametric tests between monocular acuity in the better eye and binocular acuity; Spear-man correlation analysis between monocular acuity in the better eye, binocular acuity and reading acuity, respectively.

\section{Results}

\subsection{Correlations and differences between monocular acuity in the better eye and binocular acuity}

Binocular acuity and monocular acuity in the better eye were positively correlated $(r=0.920, P<0.001)$ ( Fig.1 ) , showed by Spear-man correlation analysis.With the increase of monocular acuity in the better eye,binocular acuity was also improved. The median best corrected binocular acuity of this sample of 2520 participants is 0.6 , and the median monocular acuity in the better eye is 0.5 which reduced one line.Inter-quartile ranges of both monocular acuity in the better eye and binocular acuity were 0.5 . The difference between monocular acuity in the better eye and binocular acuity was statistically significant $(\mathrm{Z}=-23.025, \mathrm{P}<0.05)$ at the level of $\mathrm{a}=0.05$, and the former is higher.

There were 1004 cases whose binocular acuity was improved compared with monocular acuity in the better eye which was accounting for $64.61 \%$ of 1554 participants with monocular acuity equaled to 0.1 or better, thereinto, 946 improved one line according to the Standard Logarithmic Visual Acuity Chart, two and three line were 50 and 8, separately; 539 were unchanged which was accounting for 34.68\%; Decliners of binocular acuity were accounting for $0.71 \%$ with 11 cases. There were no sex and age differences above.

2.2 Correlation analysis compares reading acuity to monocular acuity in the better eye and binocular acuity

There was a linear correlation between monocular acuity in the better eye, binocular acuity and reading acuity showed by Spear-man correlation analysis, which correlation coefficients were $\mathrm{r}=0.827$, $\mathrm{P}<0.001$ and $\mathrm{r}=0.862, \quad \mathrm{P} \quad<0.001 \quad$ (Fig.2, Fig.3)respectively, the correlation coefficients were statistically significant in the test level of $a=0.05$. Correlation between binocular acuity and reading acuity was better.

\subsection{Varying degrees of visual impairment effect on reading visual acuity}

The visual acuity was divided into three groups, and participants who were unable to read in each group was counted (Table 1). Spear-man correlation 
analysis compares reading visual acuity to monocular acuity in the better eye and binocular acuity in each group of the three: correlation coefficients of group A were $\mathrm{r}=0.816, \quad \mathrm{P}<0.00$ and $\mathrm{r}=0.843, \quad \mathrm{P}<0.001$ (Fig.A $A_{1}$,Fig. $A_{2}$ ); group $B$ were $r=0.631, P<0.001$ and $\mathrm{r}=0.718, \quad \mathrm{P}<0.001\left(\right.$ Fig. $\mathrm{B}_{1}$, Fig. $\left.\mathrm{B}_{2}\right)$; group $\mathrm{C}$ were $\mathrm{r}=0.402, \quad \mathrm{P}<0.001$ and $\mathrm{r}=0.454, \quad \mathrm{P}<0.001$, the correlation coefficients were statistically significant in the test level of $a=0.05$. Correlation analysis compared reading acuity to either monocular acuity

\begin{tabular}{|c|c|c|c|c|c|}
\hline Groups & $\begin{array}{c}\text { Monocular } \\
\text { acuity in the } \\
\text { better eye } \\
\text { visual acuity }\end{array}$ & $\begin{array}{c}\text { Average } \\
\text { subjects }\end{array}$ & $\begin{array}{c}\text { Subjects who } \\
\text { were unable to } \\
\text { read }\end{array}$ & $\begin{array}{c}\text { The proportions of } \\
\text { those who were } \\
\text { unable to read }\end{array}$ \\
\hline Group A & $\leq 0.8, \geq 0.3$ & $0.56 \pm 0.04$ & 1148 & 17 & $1.5 \%$ \\
\hline Group B & $<0.3, \geq 0.1$ & $0.15 \pm 0.07$ & 406 & 66 & $16.3 \%$ \\
\hline Group C & $<0.1$ & $0.05 \pm 0.05$ & 103 & 82 & $79.6 \%$ \\
\hline
\end{tabular}

Table 1 groups of monocular acuity in the better eye and the frequency of who were unable to read.

\section{Discussion}

Generally speaking,vision with two eyes were different from vision with one eye in visual acuity. ${ }^{3}$ Numerous studies have demonstrated that binocular acuity was superior to the better eye monocular acuity under certain conditions. ${ }^{3-6}$ From the results of this experiment,compared with monocular acuity in the better eye, binocular acuity improved accounted for $64.61 \%$ of 1554 participants (range from 0.1 to 0.8 in the better seeing eye), $34.68 \%$ were unchanged, $0.71 \%$ were decreased. Binocular vision is better than monocular vision, not only the superimposition of the two eyes, reduced the sensory threshold, expanded the visual field, eliminated the blind spot, but produced a stereoscopic vision. ${ }^{7}$ Moreover, optimal binocular vision provides more information for keeping balance than monocular vision for less body sway length and area. ${ }^{8}$ However, binocular vision is a double-edged sword, if problems occurred to binocular vision,monocular visual symptoms would be happened that had never been, such as diplopia, anomalous retinal correspondence,stereoscopic vision loss, visual spatial bending and visual fatigue etc. ${ }^{7}$

The study of Gary S. Rubin et al. showed that the binocular acuity of older individuals aged 65 to 84 years could be inferred from a measure of monocular acuity. There was little evidence for binocular inhibition when the monocular acuity in the two eyes were unequal. ${ }^{9}$ This research suggested that binocular acuity and the better eye monocular acuity had a high correlation $(\mathrm{r}=0.920, \mathrm{P}<0.001)$; both of them could evaluate reading acuity $(\mathrm{r}=0.827, \mathrm{P}<0.001 ; \mathrm{r}=0.862, \mathrm{P}$ $<0.001$ ), binocular acuity exhibited higher correlation than monocular acuity in the better eye.Also, there were differences between monocular acuity in the better eye and binocular acuity $(\mathrm{Z}=-23.025, \mathrm{P}<0.05)$ at the level of $a=0.05$. For a significant portion of this elderly population, binocular performance was not well represented by better eye monocular measures. ${ }^{10}$ in the better eye or binocular acuity was highly correlated. The proportions of those who were unable to read increased with the serious of visual impairment, and correlations were reduced, reading acuity became worse. There were no differences of reading speed in visual impairment individuals whose acuity were more than or equal to 0.3 ; however , reading speed in visual disability (visual acuity was less than 0.3) individuals was slowed down obviously.

This indicated that to get a true sense of an individual's vision function in daily life, one must measure vision binocularly. Reading is a widely expressed goal of patients with vision loss. ${ }^{11}$ In this study, participants whose monocular acuity in the better eye superior or equal to 0.3 , whether used the best corrected acuity or uncorrected acuity were unable to read accounted for $1.5 \%$; six subjects could not read by the best corrected acuity, but they could read by uncorrected acuity, which account for $0.5 \%$ of the total. Participants in the secondary low vision (superior or equal to 0.1 and under 0.3 in the better seeing eye) could not read by the best corrected acuity, but they could read by uncorrected acuity, which account for $1 \%$. Majority of the participants in this situation said that they were not suitable with both eye opened at the same time, even if their binocular acuity were good, they could not see things for a long time,but could not read. The test of reading acuity was performed in subjects wearing glasses by the best corrected acuity. When monocular acuity corrected to the best, anisometropia values in most subjects who could not read used glasses were higher than 4D. The maximum was -16.00DS, although both binocular acuity and monocular acuity in the better eye were 0.8 after correction, there appeared symptoms of dizziness and visual distortion. When anisometropia of two eye higher than $2.50 \mathrm{D}$, which would produce retinal disparity that our brain could not tolerate, patients wearing glasses would be uncomfortable by the best corrected acuity monocularly. ${ }^{12}$ Generally,binocular visual function was good within 4D of anisometropia. If the anisometropia higher than $4 \mathrm{D}$ or $6 \mathrm{D}$, they could not be corrected by frame glasses, ${ }^{13}$ especially superior $6 \mathrm{D} .{ }^{14}$ There were also some subjects could not read because they did not having the habit of wearing glasses. There was no individual differences of reading speed in visual acuity superior or equal to 0.3 , this was agreed with a research that reduced distance visual acuity and myopia are not generally associated 
with reading difficulties. ${ }^{15}$ With decreased of the visual acuity,reading speed was reduced significantly for participants of visual disability (the better seeing eye was under 0.3 ).

Although binocular acuity appears to be the most intuitively appropriate measure of a person's vision (because the person functions with both eyes open), in the clinical and research environment, monocular acuity has been measured routinely, and different criteria have been used to measure visual impairment. These criteria included the Committee on Medical Rating of Physical Impairment of the AMA,in which Impairment of the Visual System equals ( $3 \times$ better eye value+worse eye value)/4. Although the better and worse eye values were designed to incorporate ocular motility, peripheral visual fields, and distance and near visual acuity, $\log ^{\mathrm{MAR}}$ distance visual acuity scores were typically used. A more recent measure used by the U.S. Social Security Administration to determine disability is visual acuity in the better seeing eye, as a measure of binocular function. Studies have considered that both monocular acuity in the better and binocular acuity good eye and poor eye should be involved in disability burden and visual function assessment. ${ }^{16}$ From this study, we demonstrated that binocular acuity should be considered a primary measure of visual impairment in clinical or research settings, because it better equated the state in which the person usually functions.

\section{References}

1) Azen S P, Varma R, Preston-Martin S, et al. Binocular visual acuity summation and inhibition in an ocular epidemiological study: the Los Angeles Latino Eye Study[J]. Investigative Ophthalmology and Visual Science, 2002, 43(6): 1742-1748.

2) Social Security Administration: Social Security Online. Social Security Agency Web Site, available at www.ssa.gov (last accessed August 2001).

3) Cagenello R, Halpern D L, Arditi A. Binocular enhancement of visual acuity[J]. JOSA A, 1993, 10(8): 1841-1848.

4) Pradhan S. A comparison of binocular summation in young and older patients. Curr Eye Res. 1996;15:315-319.

5) Home R. Binocular summation: a study of contrast sensitivity,

6) visual acuity, and recognition. Vision Res. 1978;18:579-585.

7) Pradhan S, Elliott DB. Clinical measurements of binocular summation and inhibition in patients with cataract. Clin Vis Sci. 1991;6:355-359.

8) Guangji Wang. Binocular Vision [M]. People's Health Publishing House, 2004.

9) $\mathrm{Wu} \mathrm{K} \mathrm{T}$, Lee G S. Influences of monocular and binocular vision on postural stability[J]. Journal of vestibular research: equilibrium \& orientation, 2015, 25(1): 15-21.

10) Rubin G S, Munoz B, Bandeen-Roche K, et al. Monocular versus binocular visual acuity as measures of vision impairment and predictors of visual disability[J]. Investigative ophthalmology \& visual science, 2000, 41(11): 3327-3334.

11) Schneck M E, Haegerstöm-Portnoy G, Lott L A, et al. Monocular vs. Binocular Measurement of Spatial Vision In Elders[J]. Optometry and vision science: official publication of the American Academy of Optometry, 2010, 87(8): 526.

12) Colenbrander A. Reading acuity - an important parameter of reading performance[C]//International Congress Series. Elsevier, 2005, 1282: 487-491.

13) Yuliang Wang. Optometry [M]. People's military medical press, 2008.

14) Ying Qi,Yuehua Zhou. Effect of Anisometropia and its Correction to Binocular Vision[J].Journal of Capital Medical University,2008, 29(6):762-765.

15) Lin Li,Shengli Sun,Wei Lu.Clinical Observation on myopic anisometropia and binocular vision[J].Ophthalmology,2006, 15(5):324-326

16) Grisham J D, Simons H D. Refractive error and the reading process: a literature analysis[J]. Journal of the American Optometric Association, 1986.

17) Consultation on development of standards for characterization of vision loss and visual functioning: Geneva, 4-5 September 2003[M]. World Health Organization, 2003. 
Fig. 1

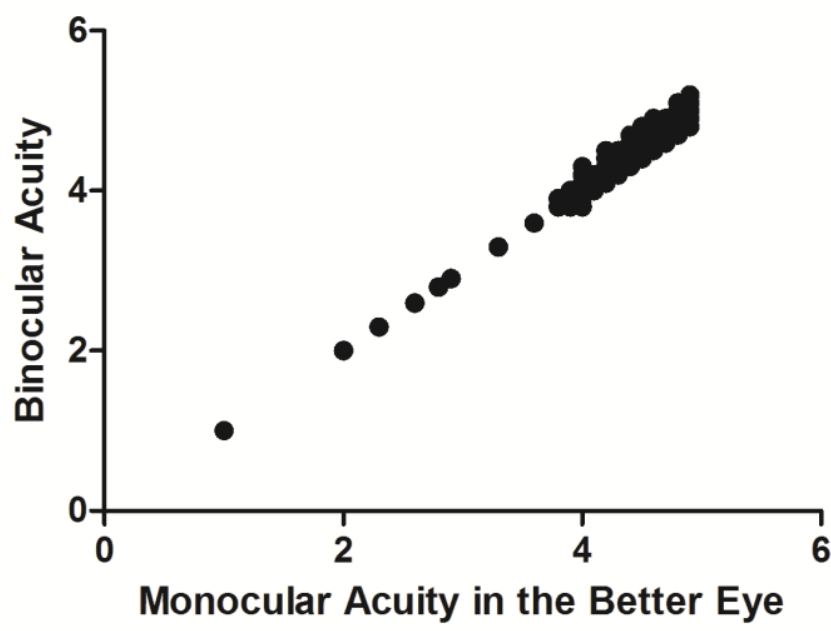

Fig. 2

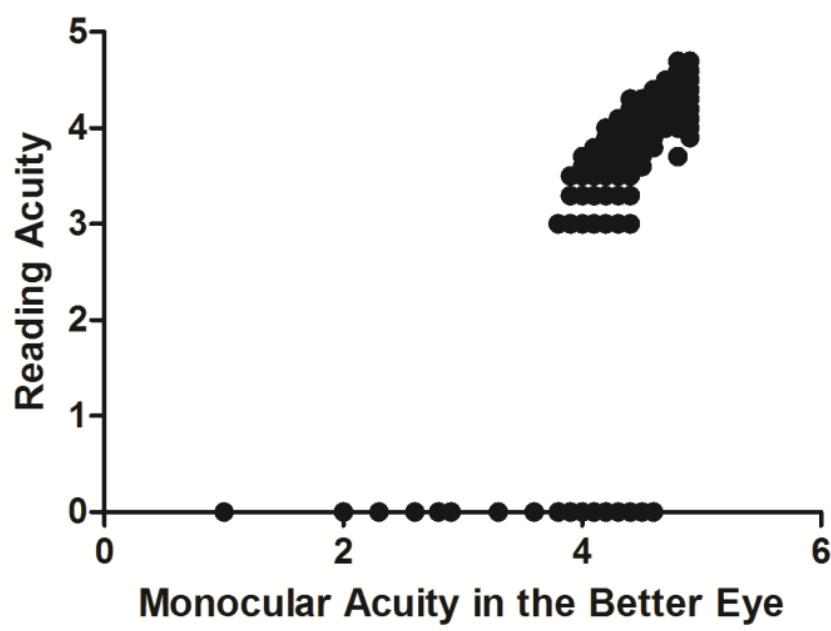

Fig. 3

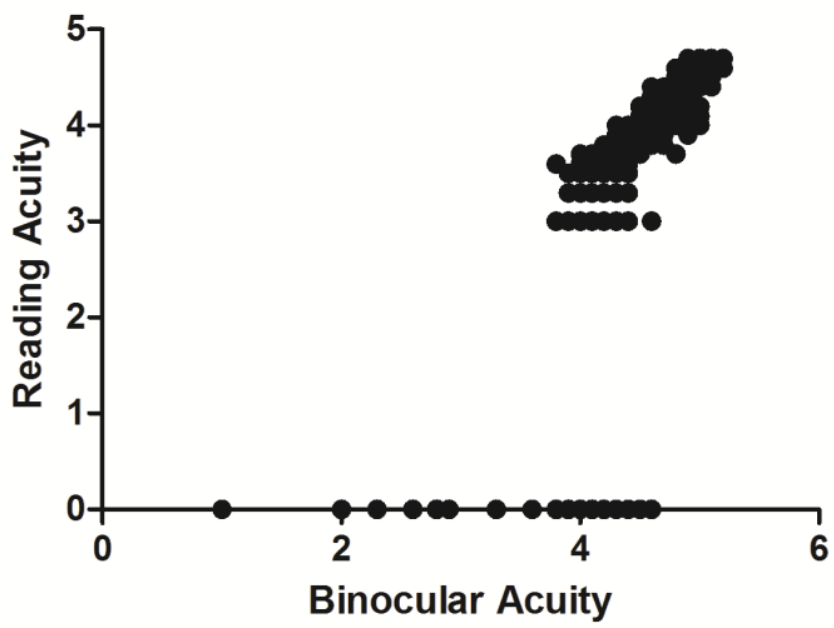


Fig. $A_{1}$

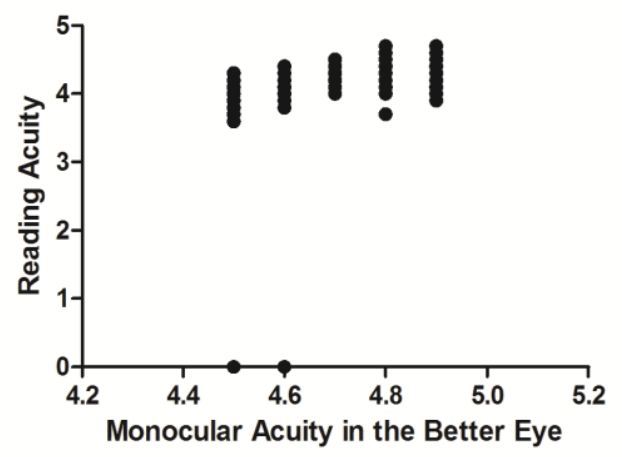

Fig. $\mathrm{B}_{1}$

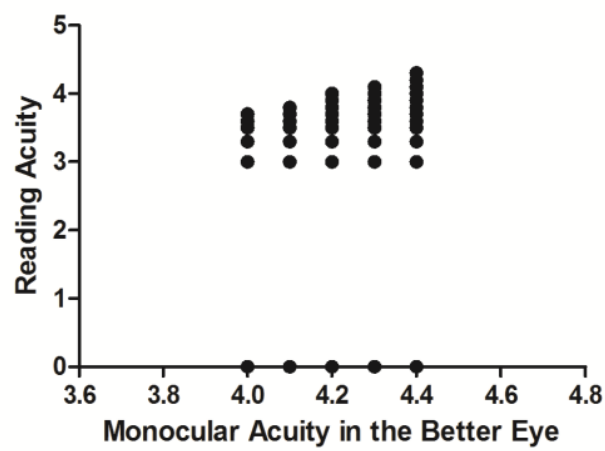

Fig. $A_{2}$

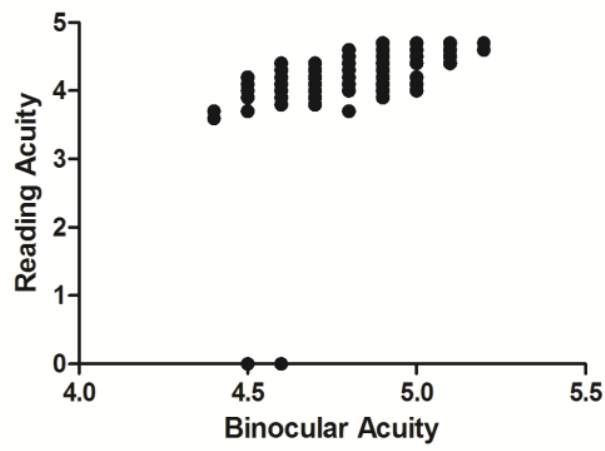

Fig. $B_{2}$

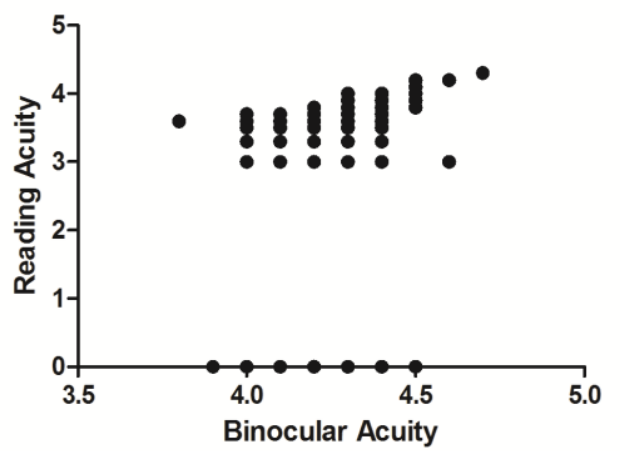

